\title{
Explicit formulas for the generalized Hermite polynomials in superspace
}

\author{
Patrick Desrosiers* \\ Département de Physique, de Génie Physique et d'Optique, \\ Université Laval, \\ Québec, Canada, G1K 7P4. \\ Luc Lapointe ${ }^{\dagger}$ \\ Instituto de Matemática y Física, \\ Universidad de Talca, \\ Casilla 747, Talca, Chile. \\ Pierre Mathieu ${ }^{\ddagger}$ \\ Département de Physique, de Génie Physique et d'Optique, \\ Université Laval, \\ Québec, Canada, G1K 7P4.
}

September 2003

\begin{abstract}
We provide explicit formulas for the orthogonal eigenfunctions of the supersymmetric extension of the rational Calogero-Moser-Sutherland model with harmonic confinement, i.e., the generalized Hermite (or Hi-Jack) polynomials in superspace. The construction relies on the triangular action of the Hamiltonian on the supermonomial basis. This translates into determinantal expressions for the Hamiltonian's eigenfunctions.
\end{abstract}

\footnotetext{
*pdesrosi@phy.ulaval.ca

†lapointe@inst-mat.utalca.cl

${ }^{\ddagger}$ pmathieu@phy.ulaval.ca
} 


\section{Introduction}

The supersymmetric extension of the Calogero-Moser-Sutherland (CMS) model [1 has been obtained some thirteen years ago, first in its rational version with confinement [2, and then a few years later in its trigonometric form [3]. It took more than ten years before a single example of an eigenfunction (for an arbitrary number of variables) could be displayed for either model [4], in which case the results relied on a special feature of the rational model, namely the existence of a transformation to a set of decoupled supersymmetric oscillators.

This low progress is to be ascribed to the lack of a number of crucial tools: 1) a general characterization of the symmetry properties of the eigenfunctions; 2) a proper labelling of the eigenfunctions; 3 ) the knowledge of a simple basis in terms of which the eigenfunctions could be expanded. It is only quite recently that the relevant tools have been designed [5]. In that work, a systematic procedure for constructing the eigenfunctions of the supersymmetric trigonometric (written stCMS) model was presented and a number of solutions were displayed. In a second step, these results were substantially improved by the explicit construction of the eigenfunctions 6. Finally, the way to linearly combine these eigenfunctions so as to form a set of orthogonal eigenfunctions was given in [7. The resulting eigenfunctions were dubbed Jack superpolynomials or equivalently, Jack superpolynomials in superspace. The superspace referred to is the euclidian space of $N$ commuting variables (the usual 'positions' in the CMS model) augmented by the $N$ anticommuting variables generated by the supersymmetrization. ${ }^{1}$

Having completely solved the trigonometric case, we then turned our attention to the rational model. A first construction of its eigenfunctions, called the generalized Hermite superpolynomials, was presented in [9]. It is the supersymmetric extension of Lassalle's result [10] in that it relates the generalized Hermite superpolynomial $J_{\Lambda}^{\omega}$ to the Jack superpolynomial $J_{\Lambda}$ (where $\Lambda$ is a superpartition and $\omega$ stands for the confining coefficient - see section 2) through the relation

$$
J_{\Lambda}^{\omega}=e^{-\Delta / 4 \omega} J_{\Lambda}
$$

Here $\Delta$ is a certain differential operator of degree minus 2 in the commuting variables. Although very elegant, this construction does not appear, a priori, to be a practical way of constructing the generalized Hermite superpolynomials. For instance, simply generating the (ordinary) polynomial $J_{(3,2,1)}^{\omega}$ requires the calculation of $\Delta^{3} J_{(3,2,1)}$. Moreover, this kind of computation has the disadvantage of depending on the number $N$ of variables in each of the two sets (commuting and anticommuting). In addition, it does not directly express the $J_{\Lambda}^{\omega}$ 's in a given basis of symmetric functions, e.g., the supermonomials $m_{\Lambda}$ or Jack polynomials $J_{\Lambda}$.

In this article, we present a completely different construction of these eigenfunctions. We essentially apply the scheme used in [6] and thereby obtain explicit expressions for the $J_{\Lambda}^{\omega}$ 's in terms of supermonomials. This method is exposed in full generality in appendix A (which can be read independently of this article). The underlying idea is that there is a road to the construction of the eigenfunctions whose central step is the solution of a very simple two-body problem. The motivation for this work was to present a practical construction of the srCMS model (i.e., free of the previously mentioned drawbacks of the method exposed in [9]) and at the same time to advertise the power of this general scheme.

The article is organized as follows. We first review briefly some (super)material needed for our construction. In section 3, we evaluate the action of the Hamiltonian on the supermonomial basis $m_{\Lambda}$. The calculation is broken down into two parts: we first evaluate the action of the Hamiltonian in the one- or two-particle sector (the former case taking care of the diagonal part of the Hamiltonian) and then extend the result to a general number of particles by adding the right symmetry factors. Breaking down the computation in this manner is obviously the only way

\footnotetext{
${ }^{1}$ These works are surveyed in [8].
} 
of unravelling a clear pattern for the action of the Hamiltonian on the supermonomials and, in the end, for the triangular decomposition of the generalized Hermite superpolynomials in terms of Jack superpolynomials. Each coefficient $c_{\Lambda, \Omega}$ can thus be written as a sum of terms, each dressed by their respective symmetry factor.

Once the action of srCMS Hamiltonian on the supermonomials is completely fixed, all the data necessary for computing the generalized Hermite superpolynomials are known. In Section 4, we end up with a tri- determinantal expression for generalized Hermite superpolynomials in terms of the supermonomials. We stress that even in the zero-fermion sector, the resulting expressions are new (albeit implicit in [10]). ${ }^{2}$

The appendix B completes the article by providing the determinantal formulas related to the Jack superpolynomials. From a computational view point, this essentially summarizes the results obtained in Refs 6, 7].

\section{Definitions}

The supersymmetric gauge transformed Hamiltonian of the srCMS model [2] reads:

$$
\mathcal{H}=2 \omega \sum_{i=1}^{N}\left(x_{i} \partial_{i}+\theta_{i} \partial_{\theta_{i}}\right)-\sum_{i=1}^{N} \partial_{i}^{2}-2 \beta \sum_{1 \leq i<j \leq N} \frac{1}{x_{i j}}\left(\partial_{i}-\partial_{j}\right)+2 \beta \sum_{1 \leq i<j \leq N} \frac{1}{x_{i j}^{2}}\left(\theta_{i j} \theta_{i j}^{\dagger}\right),
$$

where

$$
\partial_{i}=\partial_{x_{i}}, \quad x_{i j}=x_{i}-x_{j}, \quad \theta_{i j}=\theta_{i}-\theta_{j} \quad \text { and } \quad \theta_{i j}^{\dagger}=\partial_{\theta_{i}}-\partial_{\theta_{j}} .
$$

The $\theta_{i}$ 's, with $i=1, \cdots, N$, are anticommuting (Grassmannian or fermionic) variables. The commuting (bosonic) variables $x_{j}$ as well as the parameters $\beta$ and $\omega$ belong to the real field. It is easy to verify that the term $1-\theta_{i j} \theta_{i j}^{\dagger}$ is a fermionic exchange operator [3]

$$
\kappa_{i j} \equiv 1-\theta_{i j} \theta_{i j}^{\dagger}=1-\left(\theta_{i}-\theta_{j}\right)\left(\partial_{\theta_{i}}-\partial_{\theta_{j}}\right),
$$

whose action on a function $f\left(\theta_{i}, \theta_{j}\right)$ is

$$
\kappa_{i j} f\left(\theta_{i}, \theta_{j}\right)=f\left(\theta_{j}, \theta_{i}\right) \kappa_{i j} .
$$

The Hamiltonian $\mathcal{H}$ is Hermitian with respect to the following 'physical scalar product':

$$
\langle F(x, \theta), G(x, \theta)\rangle_{\beta, \omega}=\prod_{j}\left(\int_{-\infty}^{\infty} d x_{j} \int d \theta_{j} \theta_{j}\right) \prod_{k \leq l}\left(x_{k l}\right)^{2 \beta} e^{-\omega\|x\|^{2}} F(x, \theta)^{*} G(x, \theta),
$$

where $F$ and $G$ are arbitrary functions and where $\|x\|^{2}=\sum_{i} x_{i}^{2}$. The complex conjugation $*$ is defined such that

$$
\left(\theta_{i_{1}} \cdots \theta_{i_{m}}\right)^{*} \theta_{i_{1}} \cdots \theta_{i_{m}}=1 \quad \text { and } \quad x_{j}^{*}=x_{j} .
$$

In other words, $\theta_{j}^{*}$ behaves as $\theta_{j}^{\dagger}=\partial_{\theta_{j}}$. The integration over the Grassmannian variables is the standard Berezin integration, i.e.,

$$
\int d \theta=0, \quad \int d \theta \theta=1 .
$$

$$
\text { [12. }
$$

${ }^{2}$ For more details concerning the generalized Hermite polynomials without fermions, see also [1] and 
The Hamiltonian $\mathcal{H}$ preserves the space, $P^{S_{N}}$, of symmetric superpolynomials invariant under the simultaneous action of $\kappa_{i j}$ and $K_{i j}$, where $K_{i j}$ is the exchange operator acting on the $x_{i}$ variables:

$$
K_{i j} f\left(x_{i}, x_{j}\right)=f\left(x_{j}, x_{i}\right) K_{i j} .
$$

A polynomial $f$ thus belongs to $P^{S_{N}}$ if it is invariant under the action of the product

$$
\mathcal{K}_{i j}=\kappa_{i j} K_{i j},
$$

that is, if $\mathcal{K}_{i j} f=f$ for all $i, j$.

The appropriate labelling for symmetric superpolynomials is provided by superpartitions [5]. We recall that a superpartition $\Lambda$ in the $m$-fermion sector is a sequence of non-negative integers composed of two standard partitions separated by a semicolon:

$$
\Lambda=\left(\Lambda^{a} ; \Lambda^{s}\right)=\left(\Lambda_{1}, \ldots, \Lambda_{m} ; \Lambda_{m+1}, \ldots, \Lambda_{N}\right),
$$

where $\Lambda_{i}>\Lambda_{i+1} \geq 0$ for $i=1, \ldots m-1$ and $\Lambda_{j} \geq \Lambda_{j+1} \geq 0$ for $j=m+1, \ldots, N-1$. In the zero-fermion sector, the semicolon is usually omitted and $\Lambda$ reduces then to $\Lambda^{s}$. We denote the degree of a superpartition and its fermionic number respectively by:

$$
|\Lambda|=\sum_{i=1}^{N} \Lambda_{i}, \quad \text { and } \quad \underline{\bar{\Lambda}}=m .
$$

The partition rearrangement in non-increasing order of the entries of $\Lambda$ is denoted $\Lambda^{*}$.

This allows to define dominance (partial) ordering on superpartitions. We recall the usual dominance ordering on two partitions $\lambda$ and $\mu$ of the same degree: $\lambda \leq \mu$ iff $\lambda_{1}+\ldots+\lambda_{k} \leq$ $\mu_{1}+\ldots+\mu_{k}$ for all $k$. The dominance ordering on superpartitions is similar:

$$
\Omega \leq \Lambda \quad \text { if either } \Omega^{*}<\Lambda^{*} \quad \text { or } \quad \Omega^{*}=\Lambda^{*} \quad \text { and } \quad \Omega_{1}+\ldots+\Omega_{k} \leq \Lambda_{1}+\ldots \Lambda_{k}, \quad \forall k .
$$

We now furnish two fundamental bases in the space $P^{S_{N}}$. The simplest one is the monomial basis, denoted $\left\{m_{\Lambda}\right\}_{\Lambda}$, with $m_{\Lambda} \equiv m_{\Lambda}(x, \theta)$ a superanalogue of a monomial symmetric function defined as follows [5]:

$$
m_{\Lambda}=\sum_{\sigma \in S_{N}}^{\prime} \theta^{\sigma(1, \ldots, m)} x^{\sigma(\Lambda)}
$$

where the prime indicates that the summation is restricted to distinct terms, and where

$$
x^{\sigma(\Lambda)}=x_{1}^{\Lambda_{\sigma(1)}} \cdots x_{m}^{\Lambda_{\sigma(m)}} x_{m+1}^{\Lambda_{\sigma(m+1)}} \cdots x_{N}^{\Lambda_{\sigma(N)}} \quad \text { and } \quad \theta^{\sigma(1, \ldots, m)}=\theta_{\sigma(1)} \cdots \theta_{\sigma(m)} .
$$

Equivalently, we can define the supermonomials as

$$
m_{\Lambda}=\frac{1}{f_{\Lambda}} \sum_{\sigma \in S_{N}} \mathcal{K}_{\sigma}\left(\theta_{1} \cdots \theta_{m} x^{\Lambda}\right), \quad f_{\Lambda}=f_{\Lambda^{s}}=n_{\Lambda^{s}}(0) ! n_{\Lambda^{s}}(1) ! n_{\Lambda^{s}}(2) ! \cdots
$$

where $n_{\Lambda^{s}}(i)$ indicates the number of $i$ 's in $\Lambda^{s}$, the symmetric part of $\Lambda=\left(\Lambda^{a} ; \Lambda^{s}\right)$, and where $\mathcal{K}_{\sigma}$ stands for $\mathcal{K}_{i_{1}, i_{1}+1} \cdots \mathcal{K}_{i_{n}, i_{n}+1}$ when the element $\sigma$ of the symmetric group $S_{N}$ is written in terms of elementary transpositions, i.e., $\sigma=\sigma_{i_{1}} \cdots \sigma_{i_{n}}$. 
The second basis, denoted $\left\{J_{\Lambda}\right\}_{\Lambda}$, is that of the superanalogues of the Jack polynomials [7]. These superpolynomials are orthogonal and triangular eigenfunctions of the gauged stCMS Hamiltonian $\mathcal{H}_{2}$. More precisely, the Jack polynomials in superspace $J_{\Lambda} \equiv J_{\Lambda}(x, \theta ; 1 / \beta)$ are the unique functions in $P^{S_{N}}$ such that

$$
J_{\Lambda}=m_{\Lambda}+\sum_{\Omega<\Lambda} t_{\Lambda, \Omega}(\beta) m_{\Omega} \quad \text { and } \quad\left\langle J_{\Lambda}, J_{\Omega}\right\rangle_{\beta} \propto \delta_{\Lambda, \Omega},
$$

where the physical scalar product of the stCMS model is defined by

$$
\langle A(x, \theta), B(x, \theta)\rangle_{\beta}=\prod_{j}\left(\frac{1}{2 \pi i} \oint \frac{d x_{j}}{x_{j}} \int d \theta_{j} \theta_{j}\right)\left[\prod_{k \neq l}\left(1-\frac{x_{k}}{x_{l}}\right)^{\beta} A(x, \theta)^{*} B(x, \theta)\right],
$$

The variable $x_{j}$ represents the $j^{\text {th }}$ particle's position on the unit circle in the complex plane, and is thus such that $x_{j}^{*}=1 / x_{j}$.

We finally give two distinct types of eigenfunctions of the srCMS Hamiltonian $\mathcal{H}$ : the nonhomogenous supermonomials $m_{\Lambda}^{\omega}$ and the generalized Hermite superpolynomials $J_{\Lambda}^{\omega}$. . They are ' $\omega$-deformations' of the supermonomials and the Jack superpolynomials respectively, in the sense that

$$
\lim _{\omega \rightarrow \infty} m_{\Lambda}^{\omega}=m_{\Lambda} \quad \text { and } \quad \lim _{\omega \rightarrow \infty} J_{\Lambda}^{\omega}=J_{\Lambda} .
$$

The set of superpolynomials $\left\{m_{\Lambda}^{\omega}\right\}_{\Lambda}$, where $m_{\Lambda}^{\omega}=m_{\Lambda}(x, \theta ; \beta, \omega)$, is the unique basis of $P^{S_{N}}$ satisfying

$$
m_{\Lambda}^{\omega}=m_{\Lambda}+\sum_{\Omega<u} y_{\Lambda \Omega}(\beta, \omega, N) m_{\Omega} \quad \text { and } \quad \mathcal{H} m_{\Lambda}^{\omega}=2 \omega(|\Lambda|+\underline{\bar{\Lambda}}) m_{\Lambda}^{\omega},
$$

where the $u$-ordering is such that

$$
\Omega \leq_{u} \Lambda \quad \text { if either } \quad \Omega=\Lambda \quad \text { or } \quad|\Omega|=|\Lambda|-2 n,
$$

for $n=1,2,3, \ldots$ Note that we compare superpartitions belonging to the same fermionic sector, i.e., such that $\underline{\bar{\Lambda}}=\underline{\bar{\Omega}}$. Similarly, the generalized Hermite polynomials $J_{\Lambda}^{\omega} \equiv J_{\Lambda}(x, \theta ; 1 / \beta, \omega)$ form the unique basis of $P^{S_{N}}$ satisfying

$$
J_{\Lambda}^{\omega}=J_{\Lambda}+\sum_{\Omega<u} w_{\Lambda \Omega}(\beta, \omega, N) J_{\Omega} \quad \text { and } \quad \mathcal{H} J_{\Lambda}^{\omega}=2 \omega(|\Lambda|+\underline{\bar{\Lambda}}) J_{\Lambda}^{\omega} .
$$

However, in contradistinction with the $m_{\Lambda}^{\omega}$ 's, the $J_{\Lambda}^{\omega}$ 's are also orthogonal. Actually, $\left\{J_{\Lambda}^{\omega}\right\}_{\Lambda}$ is the only basis in $P^{S_{N}}$ having the two following properties [9]:

$$
J_{\Lambda}^{\omega}=J_{\Lambda}+\sum_{\Omega<u} w_{\Lambda \Omega}(\beta, \omega, N) J_{\Omega} \quad \text { and } \quad\left\langle J_{\Lambda}^{\omega}, J_{\Omega}^{\omega}\right\rangle_{\beta, \omega} \propto \delta_{\Lambda, \Omega} .
$$

with $\langle,\rangle_{\beta, \omega}$ defined in (6).

The explicit form of the eigenfunctions $m_{\Lambda}^{\omega}$ and $J_{\Lambda}^{\omega}$ will be obtained in the next two sections.

\section{Action of the srCMS Hamiltonian on supermonomials}

We want to compute the coefficients $c_{\Lambda \Omega}$ in the development

$$
\mathcal{H} m_{\Lambda}=e_{\Lambda} m_{\Lambda}+\sum_{\Omega<v \Lambda} c_{\Lambda \Omega} m_{\Omega}
$$


in terms of the ordering $\leq_{v}$ to be introduced in the next subsection.

Proceeding as in [6], we divide the calculation of $\mathcal{H} m_{\Lambda}$ into two parts. We first treat the special cases $N=1,2$. The central role played by the one-particle and two-particle sectors is rooted in the fundamental observation that the Hamiltonian is a sum of one and two-particle interactions. This computation will provide the core of the coefficient $c_{\Lambda \Omega}(\beta, \omega)$ appearing in (24). The consideration of the case $N>2$ will only dress them by symmetry factors.

To simplify the calculations, we introduce the following notation:

$$
\mathcal{H}=2 \omega \mathcal{A}-(\mathcal{B}+2 \beta \mathcal{C})
$$

where

$$
\mathcal{A}=\sum_{i} \mathcal{A}_{i}=\sum_{i}\left(x_{i} \partial_{i}+\theta_{i} \partial_{\theta_{i}}\right), \quad \mathcal{B}=\sum_{i} \mathcal{B}_{i}=\sum_{i} \partial_{i}^{2}
$$

and

$$
\mathcal{C}=\sum_{i<j} \mathcal{C}_{i j}=\sum_{i<j}\left[\frac{1}{x_{i j}}\left(\partial_{i}-\partial_{j}\right)-\frac{1}{x_{i j}^{2}}\left(1-\kappa_{i j}\right)\right] .
$$

Only the term $\mathcal{A}$ has a diagonal part. Its action is easily computed for all $N$ :

$$
2 \omega \mathcal{A} m_{\Lambda}=e_{\Lambda} m_{\Lambda}=2 \omega(|\Lambda|+\underline{\bar{\Lambda}}) m_{\Lambda} .
$$

\subsection{Action of the operator $\mathcal{B}$; the $U_{i}$ ladder operator}

The operator $\mathcal{B}$ acts trivially on one-particle supermonomials $m_{(; r)}=m_{(r)}=x_{1}^{r}$ and $m_{(r ;)}=\theta_{1} x_{1}^{r}$ :

$$
\mathcal{B}_{1} m_{[r]}=r(r-1) m_{[r-2]},
$$

where $[r]$ stands for $(; r)$ or $(r ;)$.

We translate the action of $\mathcal{B}_{i}$ on the supermonomial $m_{\Lambda}$ into that of a ladder operator $U_{i}$ acting on the superpartition $\Lambda$ as :

$$
U_{i} \Lambda=U_{i}\left(\Lambda_{1}, \cdots, \Lambda_{i}, \cdots, \Lambda_{N}\right)=\left(\Lambda_{1}, \cdots, \Lambda_{i}-2, \cdots, \Lambda_{N}\right) .
$$

We will denote by $\operatorname{sgn}\left(\sigma_{U_{i} \Lambda}^{a}\right)$ the sign of the permutation $\sigma_{U_{i} \Lambda}^{a}$ needed to reorder the entries of $U_{i} \Lambda$ (on the antisymmetric side) in order to have a superpartition: $\overline{U_{i} \Lambda}=\sigma_{U_{i} \Lambda}^{a}\left(U_{i} \Lambda\right)$. Notice that $\Lambda_{i}$ can either be part of $\Lambda^{a}$ or $\Lambda^{s}$, but a sign can only arise if $\Lambda_{i} \in \Lambda^{a}$.

Since $\left|\overline{U_{i} \Lambda}\right|=|\Lambda|-2$, successive applications of the $U_{i}$ operators on a given superpartition generate a specialization of the $u$-ordering (see Eq. (21)):

$$
\text { if } \quad \Omega=\overline{U_{i_{1}} \cdots \overline{U_{i_{n}} \Lambda}}, \quad \text { for some } i_{1}, \ldots, i_{n}, \quad \text { then } \quad \Omega \leq_{u} \Lambda .
$$

In the general case of $N \geq 2$, we have

$$
\mathcal{B} m_{\Lambda}=\sum_{\Omega ; \Omega=\overline{U_{i} \Lambda}} d_{\Lambda \Omega} m_{\Omega}
$$

where the sum is taken only over the different superpartitions $\Omega=\overline{U_{i} \Lambda}$, and where

$$
d_{\Lambda \Omega}= \begin{cases}\Lambda_{i}\left(\Lambda_{i}-1\right) \operatorname{sgn}\left(\sigma_{U_{i} \Lambda}^{a}\right), & \text { if } \quad i \in\{1, \ldots, m\} \\ \Lambda_{i}\left(\Lambda_{i}-1\right) n_{\Omega^{s}}\left(\Lambda_{i}-2\right), & \text { if } \quad i \in\{m+1, \ldots, N\} .\end{cases}
$$


Here $n_{\Omega^{s}}\left(\Lambda_{i}-2\right)$ is a symmetry factor (recall that $n_{\Omega^{s}}(a)$ gives the number of $a$ 's in $\Omega^{s}$ ). This non-trivial coefficient is determined using calculations similar to those presented in Section 4.2 of Ref. [6]. It corresponds to $f_{\Omega} \#(\Omega, \Lambda) / f_{\Lambda}$, where $\#(\Omega, \Lambda)$ denotes the number of distinct ways we can choose $i$ such that $\Omega=\overline{U_{i} \Lambda}$ and $f_{\Lambda}$ is the monomial coefficient defined in Eq. (16). For instance, if $\Lambda=(0 ; 2,1)$ then $\Omega=\overline{U_{2}(0 ; 2,1)}=(0 ; 1)$ has a symmetry factor $f_{\Omega} \#(\Omega, \Lambda) / f_{\Lambda}=$ $(N-2) ! \cdot 1 /(N-1) !=(N-2)$, so $d_{(0 ; 2,1)(0 ; 1)}=2(N-2)$.

\subsection{Action of the operator $\mathcal{C}$ in the two-particle sector}

The operator $\mathcal{C}$ contains the two-body interaction terms of the Hamiltonian. To compute its action in the two-particle sector, we need to distinguish three cases, characterized by their different fermionic sector.

\section{Case I}

In this case, the fermionic number is two and the supermonomial to be considered is $m_{(r, s ; 0)}$ :

$$
m_{(r, s ; 0)}=\frac{1}{f_{(r, s ; 0)}}\left(1+\mathcal{K}_{12}\right) \theta_{1} \theta_{2} x_{1}^{r} x_{2}^{s}=\theta_{1} \theta_{2}\left(x_{1}^{r} x_{2}^{s}-x_{1}^{s} x_{2}^{r}\right),
$$

for $r>s$. For the action of $\mathcal{C}$, a direct computation yields

$$
\begin{aligned}
\mathcal{C}_{12} m_{(r, s ; 0)}=\quad \frac{\theta_{1} \theta_{2}}{x_{12}}\left(x_{1} x_{2}\right)^{s-1} & \left\{r\left(x_{1}^{r-s} x_{2}+x_{1} x_{2}^{r-s}\right)-s\left(x_{1}^{r-s+1}+x_{2}^{r-s+1}\right)\right. \\
& \left.-2\left(x_{1}^{r-s} x_{2}+x_{1}^{r-s-1} x_{2}^{2}+\cdots+x_{1} x_{2}^{r-s}\right)\right\} .
\end{aligned}
$$

To proceed further, we use the following identity: ${ }^{3}$

$$
\begin{aligned}
& \left\{r\left(x_{1}^{r-s} x_{2}+x_{1} x_{2}^{r-s}\right)-s\left(x_{1}^{r-s+1}+x_{2}^{r-s+1}\right)-2\left(x_{1}^{r-s} x_{2}+x_{1}^{r-s-1} x_{2}^{2}+\cdots+x_{1} x_{2}^{r-s}\right)\right\} \\
& \quad=x_{12}\left\{-s\left(x_{1}^{r-s}-x_{2}^{r-s}\right)+\sum_{\ell=1}^{\lfloor(r-s-1) / 2\rfloor}(r-s-2 \ell)\left(x_{1}^{r-s-\ell} x_{2}^{\ell}-x_{1}^{\ell} x_{2}^{r-s-\ell}\right)\right\}(36)
\end{aligned}
$$

We then get

$$
\mathcal{C} m_{(r, s ; 0)}=\mathcal{C}_{12} m_{(r, s ; 0)}=-s m_{(r-1, s-1 ; 0)}+\sum_{\ell=1}^{\lfloor(r-s-1) / 2\rfloor}(r-s-2 \ell) m_{(r-1-\ell, s-1+\ell ; 0)} .
$$

\section{Case II}

In this case, we work in the one-fermion sector with $m_{(r ; s)}$ :

$$
m_{(r ; s)}=\frac{1}{f_{(r ; s)}}\left(1+\mathcal{K}_{12}\right) \theta_{1} x_{1}^{r} x_{2}^{s}=\theta_{1} x_{1}^{r} x_{2}^{s}+\theta_{2} x_{1}^{s} x_{2}^{r},
$$

${ }^{3}$ Similar identities are presented in [6]. Identity (36) can be proved along the same lines. The way such identities are found is rather simple however. The idea is to add an appropriate monomial to each monomial treated successively in order to be able to extract a factor $x_{12}$. For instance, for the monomial with the leading power of $x_{1}$, we have: $-s x_{1}^{r-s+1}=-s\left[x_{1}^{r-s} x_{12}+x_{1}^{r-s} x_{2}\right]$. We then collect all other terms of the form $x_{1}^{r-s} x_{2}$ in our original expression, add them to the newly produced $-s x_{1}^{r-s} x_{2}$ and proceed similarly to enforce the factorization of $x_{12}$. 
(since $\left.f_{(r ; s)}=1\right)$. Consider the action of $\mathcal{C}$ on $m_{(r ; s)}$, supposing first that $r>s$. We get

$$
\begin{gathered}
C_{12} m_{(r ; s)}=\frac{1}{x_{12}}\left(x_{1} x_{2}\right)^{s-1}\left[\theta_{1}\left(r x_{1}^{r-s} x_{2}-s x_{1}^{r-s+1}\right)-\theta_{2}\left(r x_{1} x_{2}^{r-s}-s x_{2}^{r-s+1}\right)\right. \\
\left.-\theta_{12}\left(x_{1}^{r-s} x_{2}+x_{1}^{r-s-1} x_{2}^{2}+\cdots+x_{1} x_{2}^{r-s}\right)\right] .
\end{gathered}
$$

We now focus on the $\theta_{1}$ term:

$$
\left.\mathcal{C}_{12} m_{(r ; s)}\right|_{\theta_{1}}=\frac{\theta_{1}}{x_{12}}\left(x_{1} x_{2}\right)^{s-1}\left\{r x_{1}^{r-s} x_{2}-s x_{1}^{r-s+1}-\left(x_{1}^{r-s} x_{2}+\cdots+x_{1} x_{2}^{r-s}\right)\right\} .
$$

A factor $x_{12}$ can again be factorized from the curly bracket, which can be seen using the identity

$$
\left\{r x_{1}^{r-s} x_{2}-s x_{1}^{r-s+1}-\left(x_{1}^{r-s} x_{2}+\cdots+x_{1} x_{2}^{r-s}\right)\right\}=x_{12}\left\{-s x_{1}^{r-s}+\sum_{\ell=1}^{r-s-1}(r-s-\ell) x_{1}^{r-s-\ell} x_{2}^{\ell}\right\}
$$

Reinserting the $\theta_{2}$ terms (by symmetry), we have

$$
\mathcal{C} m_{(r ; s)}=\mathcal{C}_{12} m_{(r ; s)}=-s m_{(r-1 ; s-1)}+\sum_{\ell=1}^{r-s-1}(r-s-\ell) m_{(r-1-\ell ; s-1+\ell)} \quad(r>s) .
$$

The derivation in the case $r<s$ is similar, with the role of the parts $r$ and $s$ interchanged:

$$
\mathcal{C} m_{(r ; s)}=\mathcal{C}_{12} m_{(r ; s)}=-r m_{(r-1 ; s-1)}+\sum_{\ell=1}^{s-r-1}(s-r-\ell) m_{(r-1+\ell ; s-1-\ell)} \quad(r<s) .
$$

Here, no reordering of the partition is required. The $r=s$ case is trivial:

$$
\mathcal{C} m_{(r ; r)}=\mathcal{C}_{12} m_{(r ; r)}=-r m_{(r-1 ; r-1)} .
$$

\section{Case III}

This case corresponds to the zero-fermion sector. We set $\Lambda=(; r, s)=(r, s)$, so that

$$
m_{(r, s)}=\frac{1}{f_{(r, s)}}\left(1+\mathcal{K}_{12}\right) x_{1}^{r} x_{2}^{s}=\frac{1}{f_{(r, s)}}\left(x_{1}^{r} x_{2}^{s}+x_{1}^{s} x_{2}^{r}\right) .
$$

Consider first the case where $r>s$, so that $f_{(r, s)}=1$. Since the action of $1-\kappa_{12}$ vanishes in absence of $\theta$ terms, the action of $\mathcal{C}_{12}$ is simply

$$
\begin{aligned}
\mathcal{C} m_{(r, s)}=\mathcal{C}_{12} m_{(r, s)} & =r \sum_{\ell=0}^{r-s-2} x_{1}^{r-2-\ell} x_{2}^{s+\ell}-s \sum_{\ell=0}^{r-s} x_{1}^{r-1-\ell} x_{2}^{s-1+\ell} \\
& =r \sum_{\ell=0}^{\lfloor(r-s-2) / 2\rfloor} m_{(r-2-\ell, s+\ell)}-s \sum_{\ell=0}^{\lfloor(r-s) / 2\rfloor} m_{(r-1-\ell, s-1+\ell)} \\
& =-s m_{r-1, s-1}+(r-s) \sum_{\ell=1}^{\lfloor(r-s) / 2\rfloor} m_{(r-1-\ell, s-1+\ell)}
\end{aligned}
$$

For $r=s$, we find exactly the same expression. Note that in reading the result, we must set $m_{\left(r^{\prime}, s^{\prime}\right)}=0$ if one label is negative. 


\subsection{Summarizing the action of $\mathcal{C}$; the $V_{i j}^{(\ell)}$ ladder operator}

Before giving the general action of $\mathcal{C}$, let us introduce the following nomenclature for a pair $(i, j)$ of indices associated to a superpartition $\Lambda$ :

$$
\begin{aligned}
& \text { type I : if } i, j \in\{1, \ldots, m\} \text {, } \\
& \text { type II : if } i \in\{1, \ldots, m\}, j \in\{m+1, \ldots, N\} \text {, } \\
& \text { type III : if } i, j \in\{m+1, \ldots, N\} \text {, }
\end{aligned}
$$

where $m=\underline{\Lambda}$ is the fermionic sector.

If we denote generically by $m_{[r, s]}$ the $N=2$ monomial appropriate to each of the three cases, we have obtained:

$$
\mathcal{C} m_{[r, s]}=\sum_{\ell=0}^{\Delta_{r, s}}\left[\left(1-\delta_{\ell, 0}\right) \max (r, s)-\min (r, s)-\eta \ell\right] m_{[r-1-\epsilon \ell, s-1+\epsilon \ell]}
$$

where $\epsilon=\operatorname{sgn}(r-s)$,

$$
\Delta_{r, s}=\left\{\begin{array}{ll}
\left\lfloor\frac{r-s-1}{2}\right\rfloor & \text { for type I } \\
\max (|r-s|-1,0) & \text { for type II } \\
\left\lfloor\frac{r-s}{2}\right\rfloor & \text { for type III }
\end{array},\right.
$$

and

$$
\eta=\left\{\begin{array}{ll}
2 & \text { for }(i, j) \text { of type I } \\
1 & \text { for }(i, j) \text { of type II } \\
0 & \text { for }(i, j) \text { of type III }
\end{array} .\right.
$$

The action of the operator $\mathcal{C}$ on supermonomials can be decomposed into three factors: a numerical prefactor (computed in the $N=2$ sector), a symmetry factor (see below) and the action of a second ladder operator $V_{i j}^{(\ell)}$ acting on superpartitions. Its action is analogous to that of the $S_{i j}^{(\ell)}$ operator, presented in Appendix A, pertaining to the description of the Jack superpolynomials. For $i<j$, it is defined only for $0 \leq \ell \leq \Delta_{\Lambda_{i} \Lambda_{j}}$, in which case

$$
V_{i j}^{(\ell)} \Lambda=V_{i j}^{(\ell)}\left(\Lambda_{1}, \cdots, \Lambda_{i}, \cdots, \Lambda_{j}, \cdots\right)=\left(\Lambda_{1}, \cdots, \Lambda_{i}-1-\epsilon \ell, \cdots, \Lambda_{j}-1+\epsilon \ell, \cdots\right),
$$

where $\epsilon$ is now given by

$$
\epsilon=\operatorname{sgn}\left(\Lambda_{i}-\Lambda_{j}\right) .
$$

Note that here, in contradistinction with the action of the operator $S_{i j}^{(\ell)}$, the number $\ell$ can be zero. The possible sign resulting from the reordering of $\overline{V_{i j}^{(\ell)} \Lambda}$ will be written $\operatorname{sgn}\left(\sigma_{V_{i j}^{(\ell)} \Lambda}^{a}\right)$.

We now take into account the symmetry factors. They have been evaluated in Section 4.2 of Ref. 6] for the case of Jack superpolynomials. However, such symmetry factors are universal: they can be used for any ladder operator $\mathcal{L}_{i j}^{\ell}$ whose action on a superpartition $\Lambda$ is given by $\left(\Lambda_{1}, \cdots, \Lambda_{i} \pm a-\epsilon \ell, \cdots, \Lambda_{j} \pm a+\epsilon \ell, \cdots\right)$ where $a$ is a positive integer. Consequently, combining the $N=2$ contribution (48) and the known symmetry factors, we get

$$
\mathcal{C} m_{\Lambda}=\sum_{\Omega} e_{\Lambda \Omega} m_{\Omega}, \quad e_{\Lambda \Omega}=\sum_{(i, j) \text { distinct } ; \overline{V_{i j}^{(\ell)} \Lambda}=\Omega} \tilde{e}_{\Lambda, V_{i j}^{(\ell)} \Lambda},
$$


where

$$
\tilde{e}_{\Lambda, V_{i j}^{(\ell)} \Lambda}=\left[\max \left(\Lambda_{i}, \Lambda_{j}\right)\left(1-\delta_{\ell, 0}\right)-\min \left(\Lambda_{i}, \Lambda_{j}\right)-\eta \ell\right] n\left(\Lambda_{i}-1-\epsilon \ell, \Lambda_{j}-1+\epsilon \ell\right) .
$$

The parameter $\eta$ is defined in (50) and $n(a, b)$ is given by:

$$
n(a, b)= \begin{cases}1 & \text { for } i, j \text { of type I } \\ n_{\Omega^{s}}(b) & \text { for } i, j \text { of type II } \\ n_{\Omega^{s}}(a) n_{\Omega^{s}}(b) & \text { for } i, j \text { of type III and } a \neq b \\ \frac{1}{2} n_{\Omega^{s}}(a)\left(n_{\Omega^{s}}(a)-1\right) & \text { for } i, j \text { of type III and } a=b\end{cases}
$$

where as before, $n_{\Omega^{s}}(i)$ denotes the number of $i$ 's in $\Omega^{s}$, the symmetric part of $\Omega=\overline{V_{i j}^{(\ell)} \Lambda}$.

In (53), we say that $(i, j)$ and $\left(i^{\prime}, j^{\prime}\right)$ are distinct if $\left(\Lambda_{i}, \Lambda_{j}\right) \neq\left(\Lambda_{i^{\prime}}, \Lambda_{j^{\prime}}\right)$ or if $\Lambda_{i}$ and $\Lambda_{i^{\prime}}$ belong to different constituent partitions $\Lambda^{a}$ or $\Lambda^{s}$. The sum over the pairs $(i, j)$ is required by the fact that a given term can be obtained in different ways, some of them associated to different symmetry factors. For example, let the initial and the final superpartitions be $\Lambda=(0 ; 2,1)$ and $\Omega=(0 ; 1)$ respectively. Three distinct pairs $(i, j)$ contribute in (53): $(1,2)$ for $\ell=1,(2,3)$ for $\ell=0$ and $(2,4)$ for $\ell=1$. Formulas (54) and (55) yield directly:

$$
\tilde{e}_{\Lambda, V_{12}^{(1)} \Lambda}=(N-2), \quad \tilde{e}_{\Lambda, V_{23}^{(0)} \Lambda}=-(N-2), \quad \tilde{e}_{\Lambda, V_{24}^{(1)} \Lambda}=(N-2)(N-3),
$$

so that

$$
\mathcal{C} m_{(0 ; 2,1)}=(N-2)(N-3) m_{(0 ; 1)} .
$$

In addition, this is also an example for which $\Omega$ is linked to $\Lambda$ by the 'one-body ladder operator' $U_{2},-$ cf. the example at the end of Section 3.1.

We stress that this pattern for the action of $\mathcal{C}$, which describes the interacting part of the Hamiltonian, would have been impossible to unravel without going through the $N=2$ analysis.

\subsection{Complete action of the Hamiltonian; the $v$-ordering}

Combining the above expressions for the action of $\mathcal{A}, \mathcal{B}$ and $\mathcal{C}$, we get the following compact expression for the action of the Hamiltonian:

$$
\mathcal{H} m_{\Lambda}=2 \omega(|\Lambda|+\underline{\bar{\Lambda}}) m_{\Lambda}-\sum_{\Omega ; \Omega=\overline{U_{i} \Lambda}} d_{\Lambda \Omega} m_{\Omega}-2 \beta \sum_{\Omega ; \Omega=\overline{V_{i j}^{(\ell)} \Lambda}} e_{\Lambda \Omega} m_{\Omega},
$$

where the coefficients $d_{\Lambda, \Omega}$ and $e_{\Lambda, \Omega}$ are given by (33) and (53)-(154) respectively.

The action of the srCMS Hamiltonian on the supermonomial basis induces a particular ordering on superpartitions that enters in the triangular decomposition of the generalized Hermite polynomials. Let us denote this ordering as $\leq_{v}$. We have seen that the action of $\mathcal{H}^{\omega}$ on $m_{\Lambda}$ is triangular and that the 'lower-order' superpartitions are simply those such that $\Omega=\overline{U_{i} \Lambda}$ or $\Omega=\overline{V_{i j}^{(\ell)} \Lambda}$ for some $i, j$. In the triangular decomposition of $\mathcal{H}^{\omega}$ on $m_{\Lambda}$, only terms obtained by one application of either $U_{i}$ or $V_{i j}^{(\ell)}$ can appear. It is thus natural to define the $v$-ordering $\leq_{v}$ in terms of multiple applications of these operators:

$$
\Omega \leq_{v} \Lambda \quad \text { iff } \quad \Omega=\overline{V_{I_{1}} \ldots \overline{V_{I_{n}} \Lambda}}
$$

for a given sequence $V_{I_{1}}, \ldots, V_{I_{n}}$, where $V_{I_{k}}$ stands for the ladder operator $U_{i_{k}}$ or $V_{i_{k} j_{k}}^{\left(\ell_{k}\right)}$. 
Again, the combined action of ladder operators $U_{i}$ and $V_{i j}^{(\ell)}$ on a superpartition $\Lambda$ decreases his weight $|\Lambda|$. This means that the $v$-ordering furnishes another refinement of the $u$-ordering:

$$
\text { if } \quad \Omega \leq_{v} \Lambda \quad \text { then } \quad \Omega \leq_{u} \Lambda .
$$

To summarize, the action of $\mathcal{H}$ is triangular in the supermonomial basis $\left\{m_{\Lambda}\right\}_{\Lambda}$ :

$$
\mathcal{H} m_{\Lambda}=2 \omega(|\Lambda|+\underline{\bar{\Lambda}}) m_{\Lambda}+\sum_{\Omega<{ }_{v} \Lambda} c_{\Lambda \Omega}(\beta, \omega, N) m_{\Omega},
$$

with

$$
\sum_{\Omega<{ }_{v} \Lambda} c_{\Lambda \Omega}(\beta, \omega, N) m_{\Omega}=-\sum_{\substack{\Omega ; \\ \Omega=\overline{U_{i} \Lambda}}} d_{\Lambda \Omega} m_{\Omega}-2 \beta \sum_{\Omega} \sum_{\substack{(i, j) \\ \Omega=V_{i j} V_{i j}^{(\ell)} \Lambda}} \tilde{e}_{\Lambda, V_{i j}^{(\ell)} \Lambda} m_{\Omega} .
$$

Note that two superpartitions such that $\Omega<_{v} \Lambda$ have distinct eigenvalues, that is, $e_{\Omega} \neq e_{\Lambda}$ since $|\Omega|<|\Lambda|$ in that case. This property is essential when writing the eigenfunctions as determinants.

\section{Determinantal formulas}

The preceding section contains three essential properties concerning the action of the Hamiltonian $\mathcal{H}$ on a generic supermonomial $m_{\Lambda}$ :

1. it is finite;

2. it is triangular with respect to the $v$-ordering ;

3. the coefficient $c_{\Lambda \Omega}$ of the monomial $m_{\Omega}$ appearing in the development (61) is known, that is, it can be computed via (33) and (53)-(54).

Thus, it is possible to construct an eigenfunction of $\mathcal{H}$ that has the following triangular form:

$$
m_{\Lambda}+\sum_{\Omega<v \Lambda} w_{\Lambda \Omega}(\beta, \omega, N) m_{\Omega}
$$

But, by virtue of Eqs (20) and (60), this function must be the very superpolynomial $m_{\Lambda}^{\omega}$. Moreover, the triangularity together with the property $\Omega<_{v} \Lambda \Rightarrow e_{\Omega} \neq e_{\Lambda}$ allow us to write the eigenfunction $m_{\Lambda}^{\omega}$ as a determinant. More precisely, using arguments similar to those in [15], we get the following two results.

Theorem 1. Let $\Lambda^{(1)} \prec_{v} \Lambda^{(2)} \prec_{v} \ldots \prec_{v} \Lambda^{(n)}=\Lambda$ where $\prec_{v}$ is a total ordering compatible with $<_{v}$. Then the Hamiltonian $\mathcal{H}$ has a triangular eigenfunction $m_{\Lambda}^{\omega}$ of eigenvalue $e_{\Lambda}=2 \omega(|\Lambda|+\underline{\bar{\Lambda}})$ that is given by the following determinant:

$$
m_{\Lambda}^{\omega}=W_{\Lambda}\left|\begin{array}{cccccc}
m_{\Lambda^{(1)}} & m_{\Lambda^{(2)}} & \ldots & \cdots & m_{\Lambda^{(n-1)}} & m_{\Lambda^{(n)}} \\
e_{\Lambda}^{(1)}-e_{\Lambda}^{(n)} & c_{\Lambda^{(2)} \Lambda^{(1)}} & \cdots & \cdots & c_{\Lambda^{(n-1)} \Lambda^{(1)}} & c_{\Lambda^{(n)} \Lambda^{(1)}} \\
0 & e_{\Lambda}^{(2)}-e_{\Lambda}^{(n)} & \ldots & \cdots & c_{\Lambda^{(n-1)} \Lambda^{(2)}} & c_{\Lambda^{(n)} \Lambda^{(2)}} \\
\vdots & 0 & \ddots & & \vdots & \vdots \\
\vdots & \vdots & \ddots & \ddots & & \vdots \\
0 & 0 & \cdots & 0 & e_{\Lambda}^{(n-1)}-e_{\Lambda}^{(n)} & c_{\Lambda^{(n)} \Lambda^{(n-1)}}
\end{array}\right|
$$


where the 'weight' constant of proportionality is

$$
W_{\Lambda}=(-1)^{n-1} \prod_{i=1}^{n-1} \frac{1}{e_{\Lambda}^{(i)}-e_{\Lambda}^{(n)}}
$$

Corollary 2. Let $\Lambda^{(1)} \prec_{v} \Lambda^{(2)} \prec_{v} \ldots \prec_{v} \Lambda^{(n)}=\Lambda$ where $\prec_{v}$ is a total ordering compatible with $<_{v}$. Then

$$
m_{\Lambda}^{\omega}=\sum_{k=1}^{n} w_{\Lambda \Lambda^{(k)}}(\beta, \omega, N) m_{\Lambda^{(k)}}
$$

where $w_{\Lambda \Lambda^{(n)}}=w_{\Lambda \Lambda}=1$, and where the coefficients $w_{\Lambda \Lambda^{(k)}}$ for $1<k \leq n$ satisfy the following recursion formula:

$$
w_{\Lambda \Lambda^{(k-1)}}=\frac{1}{e_{\Lambda}-e_{\Lambda}^{(k-1)}} \sum_{\ell=k}^{n} w_{\Lambda \Lambda^{(\ell)}} c_{\Lambda^{(\ell)} \Lambda^{(k-1)}} .
$$

However, the eigenfunctions $m_{\Lambda}^{\omega}$ are not orthogonal with respect to the scalar product $\langle,\rangle_{\beta, \omega}$. Furthermore, in the zero-fermion sector, the dominant term in $m_{\Lambda}^{\omega}=m_{\Lambda}+\sum_{\Omega<{ }_{v} \Lambda} w_{\Lambda \Omega} m_{\Omega}$ is not the Jack polynomial $J_{\Lambda}$. Consequently, the function $m_{\Lambda}^{\omega}$ cannot be the sought for generalized Hermite polynomial $J_{\Lambda}^{\omega}$. The appropriate way to construct the generalized Hermite polynomials in superspace is however obvious at this point: linearly combine the eigenfunctions $m_{\Gamma}^{\omega}$ sharing the same weight $|\Lambda|$ in such a way that the Jack superpolynomial $J_{\Lambda}$ is the term of homogeneous degree $|\Lambda|$ of this linear combination.

Theorem 3. Let $\leq$ be the dominance ordering on superpartitions (cf. Eq. (13)), and let $t_{\Lambda \Gamma}$ be the coefficient of $m_{\Gamma}$ in the development of the Jack polynomial $J_{\Lambda}$ given in Eq. [17). Then, the generalized Hermite polynomial in superspace $J_{\Lambda}^{\omega}$ reads

$$
J_{\Lambda}^{\omega}=m_{\Lambda}^{\omega}+\sum_{\Gamma<\Lambda} t_{\Lambda \Gamma}(\beta) m_{\Gamma}^{\omega}
$$

Proof. First, it is obvious that the expression (68) defines an eigenfunction of the Hamiltonian $\mathcal{H}$ with eigenvalue $2 \omega(|\Lambda|+\underline{\bar{\Lambda}})$. Second, let us recall that the generalized Hermite superpolynomials $J_{\Lambda}^{\omega}$ are the unique eigenfunctions of $\mathcal{H}$ in superspace such that (cf. Eq. (22)) $J_{\Lambda}^{\omega}=J_{\Lambda}+\sum_{\Omega<_{u} \Lambda} w_{\Lambda \Omega} J_{\Omega}$. But Eqs (68), (17) and (21) also imply that

$$
m_{\Lambda}^{\omega}+\sum_{\Gamma<\Lambda} t_{\Lambda \Gamma}(\beta) m_{\Gamma}^{\omega}=J_{\Lambda}+\sum_{\Gamma<{ }_{u} \Lambda} \tilde{t}_{\Lambda \Gamma}(\beta, \omega, N) J_{\Omega}
$$

for some coefficient $\tilde{t}_{\Lambda \Gamma}$. Thus, from the uniqueness property, $m_{\Lambda}^{\omega}+\sum_{\Gamma<\Lambda} t_{\Lambda, \Gamma} m_{\Gamma}^{\omega}$ must be the generalized Hermite superpolynomial $J_{\Lambda}^{\omega}$.

The equation (68) can be interpreted as a 'tri-determinantal' formula of the generalized Hermite polynomials in terms of the supermonomials $m_{\Omega}$. Why? Because the coefficients $t_{\Lambda \Omega}$ come from determinants (92) and (94) while the coefficients $w_{\Lambda \Omega}$ are obtained from determinant (64). From the computational point of view, this means that, when the monomial decomposition of a Jack superpolynomial $J_{\Lambda}$ is known (using a bi-determinantal formula), we get the exact expression of $J_{\Lambda}^{\omega}$ in the monomial basis by developing the $m_{\Gamma}^{\omega}$ 's in (68) via the determinantal formula (64). 
Before concluding this article, it might be of interest to present an example. Let us compute $J_{(0 ; 2,1)}^{\omega}$ in terms of the Jack superpolynomials using the determinantal formulas. Tables 1 and 2 in Ref. [7] (or the determinantal formulas in Appendix A) give the Jack superpolynomial

$$
J_{(0 ; 2,1)}=m_{(0 ; 2,1)}+\frac{2 \beta}{1+2 \beta} m_{\left(1 ; 1^{2}\right)}+\frac{6 \beta}{1+2 \beta} m_{\left(0 ; 1^{3}\right)} .
$$

Theorem $[$ tells us that the generalized Hermite superpolynomial associated to $\Lambda=(0 ; 2,1)$ is simply

$$
J_{(0 ; 2,1)}^{\omega}=m_{(0 ; 2,1)}^{\omega}+\frac{2 \beta}{1+2 \beta} m_{\left(1 ; 1^{2}\right)}^{\omega}+\frac{6 \beta}{1+2 \beta} m_{\left(0 ; 1^{3}\right)}^{\omega} .
$$

We next compute the eigenfunctions $m_{(0 ; 2,1)}^{\omega}, m_{\left(1 ; 1^{2}\right)}^{\omega}$ and $m_{\left(0 ; 1^{3}\right)}^{\omega}$ in the supermonomial basis using Theorem 1 Direct calculations give

$$
\begin{aligned}
& m_{(0 ; 2,1)}^{\omega}=m_{(0 ; 2,1)}-\frac{(N-2)[1+\beta(N-3)]}{2 \omega} m_{(0 ; 1)}, \\
& m_{\left(1 ; 1^{2}\right)}^{\omega}=m_{\left(1 ; 1^{2}\right)}+\frac{\beta(N-2)}{2 \omega} m_{(0 ; 1)}+\frac{\beta(N-1)(N-2)}{4 \omega} m_{(1 ; 0)}, \\
& m_{\left(0 ; 1^{3}\right)}^{\omega}=m_{\left(0 ; 1^{3}\right)}+\frac{\beta(N-2)(N-3)}{4 \omega} m_{(0 ; 1)}, \\
& m_{(1 ; 0)}^{\omega}=m_{(1 ; 0)}=J_{(1 ; 0)}-\frac{\beta}{1+\beta} J_{(0 ; 1)}, \\
& m_{(0 ; 1)}^{\omega}=m_{(0 ; 1)}=J_{(0 ; 1)} .
\end{aligned}
$$

Substituting these expansions into (71) leads to the decomposition of $J_{(0 ; 2,1)}^{\omega}$ in the undeformed supermonomial basis. Although we do not have derived in general the explicit form of the decomposition in terms of Jack superpolynomials, in this special case it can be easily checked to read as:

$$
J_{(0 ; 2,1)}^{\omega}=J_{(0 ; 2,1)}+\frac{\beta^{2}(N-1)(N-2)}{2 \omega(1+2 \beta)} J_{(1 ; 0)}-\frac{(N-2)(1+N \beta)}{2 \omega(1+\beta)(1+2 \beta)} J_{(0 ; 1)} .
$$

\section{Conclusion}

We have expressed the generalized Hermite superpolynomials, the eigenfunctions of the srCMS model, in determinantal form. This amounts to writing them in terms of the deformed supermonomial basis as

$$
J_{\Lambda}^{\omega}=m_{\Lambda}^{\omega}+\sum_{\Gamma<\Lambda} t_{\Lambda \Gamma}(\beta) m_{\Gamma}^{\omega}
$$

with known coefficients. Notice that in this decomposition, all the dependence on $N$ comes from the $m_{\Lambda}^{\omega}$ 's. This nevertheless implies that, in contradistinction with the Jack superpolynomials, the dependence on $N$ is inherent in the generalized Hermite superpolynomials. In other words, the $J_{\Lambda}^{\omega}$ 's are not stable with respect to the number of variables. ${ }^{4}$

The somewhat complicated form of the expressions resulting from this construction should not cast a shadow over the fact that all eigenfunctions of the srCMS model are thereby obtained in an explicit way.

\footnotetext{
${ }^{4}$ See also the example (73) $)$ which express a special $J_{\Lambda}^{\omega}$ in the $J_{\Lambda}$ basis; in that case, the $N$-dependence is completely captured by the coefficients.
} 


\section{A A general scheme for the explicit construction of the eigenfunctions}

A large class of generalized orthogonal symmetric functions can be characterized by an eigenvalue problem of the Calogero-Moser-Sutherland (CMS) type. More precisely, these multi-variable polynomials are eigenfunctions of a gauged CMS Hamiltonian $\mathcal{H}$, where the gauge transformation refers to the removal of the ground-state wave function $\psi_{0}$ from the genuine CMS Hamiltonian $H$ via the conjugation $\mathcal{H}=\psi_{0} H \psi_{0}^{-1}$. A crucial property of $\mathcal{H}$ is that it can be broken up as a sum of one- or two-body interaction terms, i.e., as

$$
\mathcal{H}=\sum_{i, j=1}^{N} \mathcal{H}_{i j}=\sum_{i, j=1}^{N}\left[\mathcal{H}_{i j}^{(1)}+\mathcal{H}_{i j}^{(2)}+\cdots\right],
$$

with $N$ the number of interacting particles. Here $\mathcal{H}_{i j}^{(p)}$ could be a diagonal term (e.g., a kinetic energy term) or more generally a single-body term, in which case it would be proportional to $\delta_{i j}$, or an interaction term, in which case $\mathcal{H}_{i j}^{(p)} \propto\left(1-\delta_{i j}\right)$.

There is a systematic scheme for constructing explicitly the eigenfunctions of $\mathcal{H}$ that also provides closed-form expressions. By an explicit construction, we refer to the complete specification of the expansion coefficients of the sought for eigenfunctions in a prescribed basis, typically the basis of monomial symmetric functions $m_{\lambda}$, where $\lambda$ is a partition. More precisely, we look for eigenfunctions $P_{\lambda}$ of the form

$$
P_{\lambda}=m_{\lambda}+\sum_{\mu<\lambda} \alpha_{\lambda, \mu} m_{\mu}
$$

that is, triangular with respect to a certain ordering on partitions and normalized such that $\alpha_{\lambda, \lambda}=$ 1. In short, the explicit construction of $P_{\lambda}$ refers to the determination of the coefficients $\alpha_{\lambda, \mu}$.

This scheme relies heavily on the intrinsic two-body nature of the CMS interaction. The first step amounts to treat the $N=2$ problem and evaluate explicitly the action of $\mathcal{H}_{12}$ on a generic (two-part) monomial function $m_{\lambda}=m_{(r, s)}\left(r=\lambda_{1}, s=\lambda_{2}\right)$ :

$$
\mathcal{H}_{12} m_{(r, s)}=\sum_{p} \mathcal{H}_{12}^{(p)} m_{(r, s)}=\epsilon_{(r, s)} m_{(r, s)}+\sum_{\left(r^{\prime}, s^{\prime}\right) \neq(r, s)} \sum_{p} c_{(r, s),\left(r^{\prime}, s^{\prime}\right)}^{(p)} m_{\left(r^{\prime}, s^{\prime}\right)}
$$

In the CMS case, the action actually turns out to be triangular: the ordering $<$ on partitions with two entries being fixed such that $\left(r^{\prime}, s^{\prime}\right)<(r, s)$ when $\left(r^{\prime}, s^{\prime}\right)$ appears in the sum.

Actually, to each element $\mathcal{H}_{12}^{(p)}$ in the decomposition of $\mathcal{H}_{12}$ corresponds a ladder (lowering) operator $\mathcal{L}_{12}^{(p)}$ acting on partitions. To be more precise, to any $m_{\left(r^{\prime}, s^{\prime}\right)}$ appearing in $\mathcal{H}_{12} m_{(r, s)}$ there corresponds a ladder operator that acts on the partition $(r, s)$ to produce $\left(r^{\prime}, s^{\prime}\right)$. The ordering governing the triangular decomposition can thus be defined precisely as follows: $\left(r^{\prime}, s^{\prime}\right)<(r, s)$ if there is a ladder operator $\mathcal{L}_{12}^{(p)}$ such that $\left(r^{\prime}, s^{\prime}\right)=\mathcal{L}_{12}^{(p)}(r, s)$.

This first step which consists in solving the $N=2$ case, even though clearly model-dependent, is usually rather straightforward. The second step now amounts to extending this result from $N=2$ to a general $N$, that is, to obtaining explicitly

$$
\mathcal{H} m_{\lambda}=\epsilon_{\lambda} m_{\lambda}+\sum_{\mu<\lambda} c_{\lambda, \mu} m_{\mu} .
$$

The computation of the diagonal coefficient $\epsilon_{\lambda}$ is normally quite simple. The main technical difficulty lies in the determination of the non-diagonal coefficients $c_{\lambda, \mu}$. The key point is that all 
the monomials $m_{\mu}$ that appear in this expansion can be traced back to an underlying two-body interaction. In other words, a partition $\mu$ such that $c_{\lambda, \mu} \neq 0$ differs from $\lambda$ in at most two of its entries, say the $i$-th and the $j$-th ones. This implies that $c_{\lambda, \mu}$ is determined by the corresponding two-body coefficient $c_{\left(\lambda_{i}, \lambda_{j}\right),\left(\mu_{i}, \mu_{j}\right)}^{(p)}$ up to a symmetry factor $a_{\mu}^{(p)}$ :

$$
c_{\lambda, \mu}=\sum_{p} a_{\mu}^{(p)} c_{\left(\lambda_{i}, \lambda_{j}\right),\left(\mu_{i}, \mu_{j}\right)}^{(p)} .
$$

This symmetry factor $a_{\mu}^{(p)}$ is characteristic of the generic form of $\mathcal{H}_{i j}^{(p)}$, that is, whether it is a single- or two-body term. It takes care of the different ways we can relate $\lambda$ to $\mu$; it is thus a purely combinatorial factor, solely determined by the multiplicity of the part $\mu_{i}$ in $\mu$ if it is related to a diagonal term, or the multiplicity of both $\mu_{i}$ and $\mu_{j}$ for a genuine interaction term. In that sense, the symmetry factors are universal, i.e., independent of the precise form of the Hamiltonian $\mathcal{H} .{ }^{5}$

After the completion of these first two steps, the action of $\mathcal{H} m_{\lambda}$ is known. The third step amounts to a direct construction of the eigenfunctions $P_{\lambda}$ in the form of a determinant involving the following entries: $m_{\lambda}, \epsilon_{\lambda}$ and $c_{\lambda, \mu}$. By expanding the determinant, we obtain the decomposition (76) with all $\alpha_{\lambda, \mu}$ determined. The sum runs now over all $\mu<\lambda$, where the ordering governing this triangular decomposition is precisely the ordering that underlies the decomposition of $\mathcal{H} m_{\lambda}$ (in the sense that we say $\mu<\lambda$ if $m_{\mu}$ appears in $\mathcal{H}^{n} m_{\lambda}$, for a certain value of $n$ ).

The general scheme is thus quite simple in principle and totally constructive. Explicit eigenfunctions can be worked out in this way without requiring the previous knowledge of anything more than the monomial basis. Examples of orthogonal polynomial that can be computed in this way include the Jack polynomials and the generalized Hermite, Jacobi and Laguerre polynomials. For Jack polynomials, eigenfunctions of the trigonometric CMS (tCMS) model, the above procedure is particularly simple because there is a single two-body term in the Hamiltonian that contributes to the coefficient $c_{\lambda, \mu}$ and also a single overall symmetry factor.

It is difficult to trace back the precise origin of this scheme in the literature. To our knowledge, its 'history' goes as follows. For Jack polynomials $J_{\lambda}$, it appears implicitly in Macdonald's book 13. In particular, the action of $\mathcal{H} m_{\lambda}$ is given in example 3(c) of Sect. VI.4 p. 327. In this expression we can recognize the two-body decomposition but there is no emphasis on the symmetry factor, which is hidden in the form of a summation. Therein, the following example 3(d) displays a recursion formula for the coefficients $\alpha_{\lambda, \mu}$ that can also be deduced from the determinantal expression of $J_{\lambda}$. The expression of $\mathcal{H} m_{\lambda}$ for the tCMS model, using the idea of first computing the two-body case and then lifting the result by a symmetry factor is due to Sogo [14] (but given without proof). The completion of the program by displaying the explicit determinantal expression for the eigenfunctions (in this case, the Jack polynomials) has first been done in [15]. Furthermore, the extension to all root systems was considered in [16].

The first explicit formulation of the above complete scheme appears to be that of [6] (see also Sect. 3.6-3.8 of [8] ), where it was applied to the construction of the Jack superpolynomials, defined to be eigenfunctions of the supersymmetric version of the tCSM model $[5]$.

\footnotetext{
${ }^{5}$ To be more precise, let us stress that in general, we may also have to sum over pairs $\left(\lambda_{i}, \lambda_{j}\right)$ that are combinatorially distinct in the sense of belonging to separate families, each of which giving rise to different symmetry factors (see the paragraph following (55) for an explicit example). However, the families are only determined by the structure of the partitions involved (the superpartitions in the case treated in this paper), and it thus remains true that the combinatorial factor is independent of the structure of the Hamiltonian. Note that the case we consider in this paper captures the full complexity of the generic situation described above in that a given monomial term $m_{\Omega}$ occurring in the decomposition of $\mathcal{H} m_{\Lambda}$ can be related to two different pieces of the two-body Hamiltonian, or to combinatorially distinct pairs $\left(\lambda_{i}, \lambda_{j}\right)$ associated to a given two-body term.
} 
In the absence of supersymmetry, the above construction leads directly to orthogonal polynomials. Indeed, eigenfunctions of $\mathcal{H}$ turn out to be also eigenfunctions of a whole tower of commuting charges $\left\{\mathcal{H}_{n}\right\}$, for $n=1,2, \ldots, N$, (where $\left.\mathcal{H}_{2}=\mathcal{H}\right)$ since these charges also act triangularly on the monomial basis with respect to the same ordering. However, in the supercase, it is not guaranteed that the superpolynomials, denoted $\mathcal{P}_{\Lambda}$, constructed along this scheme will be orthogonal. And actually, this is usually not the case. But the way one should linearly combine the $\mathcal{P}_{\Lambda}$ 's to form orthogonal superpolynomials is suggested by the integrability structure of the underlying physical problem. It is indeed rooted in the fact that the supersymmetric extension of any CMS model has twice as many commuting conserved charges than its corresponding non-supersymmetric version. In other words, there is an additional tower of $N$ conserved charges $\left\{\mathcal{I}_{n}\right\}$ (which vanish when the anticommuting variables are set equal to zero). One thus needs to iterate the procedure one step further: take a representative among this new set of conserved charge (in its gauged form) to play the role of $\mathcal{H}$ and replace $m_{\Lambda}$ (the supermonomial) by the eigenfunction $\mathcal{P}_{\Lambda}$ that was built in the first step using $\mathcal{H}$. The resulting eigenfunctions are then orthogonal. This is precisely the way the orthogonal Jack superpolynomials $J_{\Lambda}$ have been obtained in [7].

\section{B Determinantal formulas for Jack polynomials in super- space}

The explicit formulas for the Jack polynomials $J_{\Lambda}$ in superspace are written in terms of two bases of $P^{S_{N}}$ : the non-orthogonal set of eigenfunctions $\left\{\mathcal{J}_{\Lambda}\right\}_{\Lambda}$ and the monomial set $\left\{m_{\Lambda}\right\}_{\Lambda}$. These formulas make use of two special orderings on superpartitions.

The first partial ordering in related to the action of a ladder operator $S_{i j}^{(\ell)}$ on superpartitions. ${ }^{6}$ Its action, for $i<j$ and $\ell \geq 1$, is defined as follows:

$$
S_{i j}^{(\ell)}\left(\Lambda_{1}, \ldots, \Lambda_{i}, \ldots, \Lambda_{j}, \ldots\right)= \begin{cases}\left(\Lambda_{1}, \ldots, \Lambda_{i}-\ell, \ldots, \Lambda_{j}+\ell, \ldots\right) & \text { if } \Lambda_{i}>\Lambda_{j} \\ \left(\Lambda_{1}, \ldots, \Lambda_{i}+\ell, \ldots, \Lambda_{j}-\ell, \ldots\right) & \text { if } \Lambda_{j}>\Lambda_{i}\end{cases}
$$

Note that the result is such as given only if :

$$
\begin{aligned}
\text { I : } & i, j \in\{1, \ldots, m\} \text { and }\left\lfloor\frac{\Lambda_{i}-\Lambda_{j}-1}{2}\right\rfloor \geq \ell, \\
\text { II : } & i \in\{1, \ldots, m\}, j \in\{m+1, \ldots, N\} \text { and }\left|\Lambda_{i}-\Lambda_{j}\right|-1 \geq \ell, \\
\text { III : } & i, j \in\{m+1, \ldots, N\} \text { and }\left\lfloor\frac{\Lambda_{i}-\Lambda_{j}}{2}\right\rfloor \geq \ell,
\end{aligned}
$$

and otherwise, $S_{i j}^{(\ell)} \Lambda=\emptyset$. The $s$-ordering $\leq_{s}$ is defined by:

$$
\Omega \leq_{s} \Lambda \quad \text { iff } \quad \Omega=\overline{S_{i_{k} j_{k}}^{\left(\ell_{k}\right)} \ldots \overline{S_{i_{1} j_{1}}^{\left(\ell_{1}\right)}}}
$$

for a given sequence of operators $S_{i_{1} j_{1}}^{\left(\ell_{1}\right)}, \ldots, S_{i_{k} j_{k}}^{\left(\ell_{k}\right)}$.

Obviously, if $\Omega \leq_{s} \Lambda$ then $\Omega \leq \Lambda$, i.e., the s-ordering refines the dominance ordering. The same property holds for the second ordering on superpartitions. It is defined using the exchange operator $T_{i j}$ whose action on $\Lambda=\left(\ldots, \Lambda_{i}, \ldots, \Lambda_{j}, \ldots\right)$ is not influenced by the semi-colon and is given by :

$$
T_{i j} \Lambda= \begin{cases}\left(\ldots, \Lambda_{j}, \ldots, \Lambda_{i}, \ldots\right) & \text { if } \Lambda_{i}>\Lambda_{j} \\ \left(\ldots, \Lambda_{i}, \ldots, \Lambda_{j}, \ldots\right) & \text { otherwise }\end{cases}
$$

\footnotetext{
${ }^{6}$ The operator $S_{i j}^{(\ell)}$ reads $R_{i j}^{(\ell)}$ in $[6]$.
} 
The $t$-ordering $\leq_{t}$ is such that:

$$
\Omega \leq_{t} \Lambda \quad \text { iff } \quad \Omega=\overline{T_{i_{k} j_{k}} \ldots \overline{T_{i_{1} j_{1}} \Lambda}}
$$

for a given sequence of operators $T_{i_{1} j_{1}}, \ldots, T_{i_{k} j_{k}}$.

We are now in position to give another definition of the Jack superpolynomials in terms of the eigenvalue problem associated with two conserved operators of the stCMS model: the supersymmetric Hamiltonian $\mathcal{H}_{2}$ and the operator $\mathcal{I}_{1}$ (which does not exist in the non-supersymmetric case). Note that $\lim _{\beta \rightarrow 0} \mathcal{H}_{2}=\sum_{i}\left(x_{i} \partial_{i}\right)^{2}$ while $\lim _{\beta \rightarrow 0} \mathcal{I}_{1}=(N-1) ! \sum_{i}\left(x_{i} \partial_{i}\right)\left(\theta_{i} \partial_{\theta_{i}}\right)$.

Definition 4. The Jack polynomials $J_{\Lambda}$ in superspace are the unique functions in $P^{S_{N}}$ such that

$$
\mathcal{I}_{1} J_{\Lambda}=\epsilon_{\Lambda} J_{\Lambda} \quad \text { and } \quad J_{\Lambda}=\mathcal{J}_{\Lambda}+\sum_{\Omega<{ }_{t} \Lambda} u_{\Lambda \Omega}(\beta) \mathcal{J}_{\Omega}
$$

where the superpolynomials $\mathcal{J}_{\Lambda}$ are the unique functions such that

$$
\mathcal{H}_{2} \mathcal{J}_{\Lambda}=\varepsilon_{\Lambda} \mathcal{J}_{\Lambda} \quad \text { and } \quad \mathcal{J}_{\Lambda}=m_{\Lambda}+\sum_{\Omega<{ }_{s} \Lambda} v_{\Lambda \Omega}(\beta) m_{\Omega}
$$

The eigenvalues are given by

$$
\epsilon_{\Lambda}=(N-1) ! \sum_{i=1}^{m}\left[\lambda_{i}-\beta\left(m(m-1)+\#_{\Lambda}\right)\right]
$$

and

$$
\varepsilon_{\Lambda}=\sum_{i=1}^{N}\left[\Lambda_{i}^{2}+\beta(N+1-2 j) \Lambda\right]
$$

where $m$ stands for the fermionic sector $\underline{\bar{\Lambda}}$, while $\#_{\Lambda}$ stands for the number of pairs $(i, j)$ such that $i \in\{1, \ldots, m\}, j \in\{m+1, \ldots, N\}$ and $\Lambda_{i}<\Lambda_{j}$.

We compute the coefficients $u_{\Lambda \Omega}$ and $v_{\Lambda \Omega}$ by means of determinantal formulas (cf. [6] 7]). These formulas are written in terms of coefficients $a_{\Lambda \Omega}$ and $b_{\Lambda \Omega}$ defined by the following expressions:

$$
\mathcal{H}_{2} m_{\Lambda}=\varepsilon_{\Lambda} m_{\Lambda}+\sum_{\Omega<{ }_{s} \Lambda} a_{\Lambda \Omega}(\beta) m_{\Omega} \quad \text { and } \quad \mathcal{I}_{1} \mathcal{J}_{\Lambda}=\epsilon_{\Lambda} \mathcal{J}_{\Lambda}+\sum_{\Omega<{ }_{t} \Lambda} b_{\Lambda \Omega}(\beta, N) \mathcal{J}_{\Omega} .
$$

The coefficient $b_{\Lambda \Omega}$ reads:

$$
b_{\Lambda \Omega}= \begin{cases}(N-1) ! \beta \operatorname{sgn}\left(\sigma_{T_{i j} \Lambda}^{a}\right) n_{\Omega^{s}}\left(\Lambda_{i}\right) & \text { if } \Omega=\overline{T_{i j} \Lambda} \text { for some } i<j, \\ 0 & \text { otherwise }\end{cases}
$$

The coefficient $a_{\Lambda \Omega}(\beta)$ is more elaborated. It is non-zero only if $\Omega$ can be obtained from $\Lambda$ by a single action of the ladder operator, namely if $\Omega=\left(\Omega^{a} ; \Omega^{s}\right)=\overline{S_{i j}^{(\ell)} \Lambda}$, for a given $S_{i j}^{(\ell)}$ with $\ell>0$, in which case it reads:

$$
a_{\Lambda \Omega}(\beta)= \begin{cases}2 \beta \operatorname{sgn}\left(\sigma_{S_{i j}^{(\ell)}}^{a}\right)\left(\Lambda_{i}-\Lambda_{j}-\eta \ell\right) n\left(\Lambda_{i}-\ell, \Lambda_{j}+\ell\right) & \text { if } \Lambda_{i}>\Lambda_{j}, \\ 2 \beta \operatorname{sgn}\left(\sigma_{S_{i j}^{(\ell)}}^{a}\right)\left(\Lambda_{j}-\Lambda_{i}-\eta \ell\right) n\left(\Lambda_{i}+\ell, \Lambda_{j}-\ell\right) & \text { if } \Lambda_{j}>\Lambda_{i},\end{cases}
$$

where $\operatorname{sgn}\left(\sigma_{S_{i j}^{(\ell)}}^{a}\right)$ stands for the sign of the permutation $\sigma_{S_{i j}^{(\ell)}}^{a}$ and $\eta$ is given in (150).

Finally, the closed form expressions of the $\mathcal{J}_{\Lambda}$ 's and the $J_{\Lambda}$ 's are contained in the two following theorems. 
Theorem 5. [6] Let $\Lambda^{(1)} \prec_{s} \Lambda^{(2)} \prec_{s} \ldots \prec_{s} \Lambda^{(n)}=\Lambda$ where $\prec_{s}$ is a total ordering compatible with $<_{s}$. Then $\mathcal{J}_{\Lambda}$ is given by the following determinant:

$$
\mathcal{J}_{\Lambda}=\mathcal{E}_{\Lambda} \mid \begin{array}{cccccc}
m_{\Lambda^{(1)}} & m_{\Lambda^{(2)}} & \ldots & \cdots & m_{\Lambda^{(n-1)}} & m_{\Lambda^{(n)}} \\
\varepsilon_{\Lambda^{(1)}}-\varepsilon_{\Lambda^{(n)}} & a_{\Lambda^{(2)} \Lambda^{(1)}} & \cdots & \cdots & a_{\Lambda^{(n-1)} \Lambda^{(1)}} & a_{\Lambda^{(n)} \Lambda^{(1)}} \\
0 & \varepsilon_{\Lambda^{(2)}}-\varepsilon_{\Lambda^{(n)}} & \cdots & \cdots & a_{\Lambda^{(n-1)} \Lambda^{(2)}} & a_{\Lambda^{(n)} \Lambda^{(2)}} \\
\vdots & 0 & \ddots & & \vdots & \vdots \\
\vdots & \vdots & \ddots & \ddots & & \vdots \\
0 & 0 & \cdots & 0 & \varepsilon_{\Lambda^{(n-1)}}-\varepsilon_{\Lambda^{(n)}} & a_{\Lambda^{(n)} \Lambda^{(n-1)}}
\end{array}
$$

where the constant of proportionality is

$$
\mathcal{E}_{\Lambda}=(-1)^{n-1} \prod_{i=1}^{n-1} \frac{1}{\varepsilon_{\Lambda^{(i)}}-\varepsilon_{\Lambda^{(n)}}},
$$

Theorem 6. [7] Let $\Lambda^{(1)} \prec_{t} \Lambda^{(2)} \prec_{t} \ldots \prec_{t} \Lambda^{(n)}=\Lambda$ where $\prec_{t}$ is a total ordering compatible with $<_{t}$. Then $J_{\Lambda}$ is given by the following determinant:

$$
J_{\Lambda}=E_{\Lambda}\left|\begin{array}{cccccc}
\mathcal{J}_{\Lambda^{(1)}} & \mathcal{J}_{\Lambda^{(2)}} & \cdots & \cdots & \mathcal{J}_{\Lambda^{(n-1)}} & \mathcal{J}_{\Lambda^{(n)}} \\
\epsilon_{\Lambda^{(1)}}-\epsilon_{\Lambda^{(n)}} & b_{\Lambda^{(2)} \Lambda^{(1)}} & \cdots & \cdots & b_{\Lambda^{(n-1)} \Lambda^{(1)}} & b_{\Lambda^{(n)} \Lambda^{(1)}} \\
0 & \epsilon_{\Lambda^{(2)}}-\epsilon_{\Lambda^{(n)}} & \cdots & \cdots & b_{\Lambda^{(n-1)} \Lambda^{(2)}} & b_{\Lambda^{(n)} \Lambda^{(2)}} \\
\vdots & 0 & \ddots & & \vdots & \vdots \\
\vdots & \vdots & \ddots & \ddots & & \vdots \\
0 & 0 & \cdots & 0 & \epsilon_{\Lambda^{(n-1)}}-\epsilon_{\Lambda^{(n)}} & b_{\Lambda^{(n)} \Lambda^{(n-1)}}
\end{array}\right|
$$

where the constant of proportionality is

$$
E_{\Lambda}=(-1)^{n-1} \prod_{i=1}^{n-1} \frac{1}{\epsilon_{\Lambda^{(i)}}-\epsilon_{\Lambda^{(n)}}} .
$$

Acknowledgments. This work was supported by NSERC and FONDECYT (Fondo Nacional de Desarrollo Científico y Tecnológico) grant \#1030114. P.D. is grateful to the Fondation J.A.-Vincent for a student fellowship.

\section{References}

[1] M. A. Olshanetsky and A. M. Perelomov, Quantum integrable systems related to Lie algebras, Phys. Rept. 94 (1983), 313.

[2] D. Z. Freedman and P. F. Mende, An exactly solvable $N$ particle system in supersymmetric quantum mechanics, Nucl. Phys. B344 (1990), 317.

[3] B. Sriram Shastry and B. Sutherland, Superlax pairs and infinite symmetries in the $1 / r^{2}$ system, Phys. Rev. Lett. 70 (1993), 4029, cond-mat/9212029 L. Brink, T. H. Hansson, S. Konstein and M. A. Vasiliev, The Calogero model: Anyonic representation, fermionic extension and supersymmetry, Nucl. Phys. B401 (1993), 591, hep-th/9302023

[4] P. K. Ghosh, Super-Calogero-Moser-Sutherland systems and free super-oscillators: A mapping, Nucl. Phys. B595 (2001), 519, hep-th/0007208. 
[5] P. Desrosiers, L. Lapointe and P. Mathieu, Supersymmetric Calogero-Moser-Sutherland models and Jack superpolynomials, Nucl. Phys B606 (2001), 547, hep-th/0103178

[6] P. Desrosiers, L. Lapointe and P. Mathieu, Jack superpolynomials, superpartition ordering and determinantal formulas, Commun. Math. Phys. 233 (2003), 383, hep-th/0105107

[7] P. Desrosiers, L. Lapointe and P. Mathieu, Jack polynomials in superspace, to appear in Commun. Math. Phys., hep-th/0209074.

[8] P. Desrosiers, L. Lapointe and P. Mathieu, Supersymmetric Calogero-Moser-Sutherland models: superintegrability structure and aigenfunctions, to appear in the proceedings of the Workshop on superintegrability in classical and quantum systems, ed. P Winternitz, CRM series, Springer, hep-th/0210190

[9] P. Desrosiers, L. Lapointe and P. Mathieu, Generalized Hermite polynomials in superspace as eigenfunctions of the supersymmetric rational CMS model, to appear in Nucl. Phys. B, hep-th/0305038.

[10] M. Lassalle, Polynômes de Hermites généralisés, C.R. Acad. Sci. Paris, t. 313, séries I (1991), 579 .

[11] J. F. van Diejen, Confluent hypergeometric orthogonal polynomials related to the rational quantum Calogero system with harmonic confinement, Commun. Math. Phys. 188 (1997), 467.

[12] T. H. Baker and P. J. Forrester, The Calogero-Sutherland model and generalized polynomials Commun. Math. Phys. 188 (1997), 1175, solv-int/9608004.

[13] I. G. Macdonald, Symmetric functions and Hall polynomials, 2nd ed., Oxford University Press (1995).

[14] K. Sogo, Eigenstates of Calogero-Sutherland-Moser model and generalized Schur functions, J. Math. Phys. 35 (1994), 2282.

[15] L. Lapointe, A. Lascoux and J. Morse, Determinantal formula and recursion for Jack polynomials, Electro. J. Comb. 7 (2000), 467.

[16] J.-F. van Diejen, L. Lapointe, and J. Morse, Determinantal construction of orthogonal polynomials associated with root systems, submitted. 